\title{
Association Between Serum C1q Tumor Necrosis Factor-Related Protein 9 and the Clinical Characteristics and Prognosis of Ischemic Stroke
}

\author{
Cui Yang · Jia-yan Xin · Zhi-lan Liu · Fan Fan • Yun-ming Li • \\ Fan Jin · Qing-song Wang · Fu-qiang Guo • Neng-wei Yu • \\ Wei-dong Le · Yang Xiang (D)
}

Received: September 5, 2021 / Accepted: October 19, 2021 / Published online: November 2, 2021

(C) The Author(s) 2021

\begin{abstract}
Introduction: $\mathrm{C} 1 \mathrm{q}$ tumor necrosis factor (TNF)-related protein 9 (CTRP9) is a novel member of the C1q/TNF superfamily. According to our previous review, CTRP9 plays a vital role in the process of cardiovascular diseases, including regulating energy metabolism, modulating vasomotion, protecting endothelial cells, inhibiting platelet activation, inhibiting pathological vascular remodeling, stabilizing atherosclerotic plaques, and protecting the
\end{abstract}

Cui Yang and Jia-yan Xin contributed equally to the manuscript (co-first authors).

Supplementary Information The online version contains supplementary material available at https:// doi.org/10.1007/s40120-021-00296-7.

C. Yang · W. Le $(\bowtie) \cdot$ Y. Xiang $(\varangle)$

Institute of Neurology, Sichuan Provincial People's

Hospital, University of Electronic Science and

Technology of China, Chengdu, China

e-mail: wdle@sibs.ac.cnY.Xiang

e-mail: xiangyang2020spph@126.com

C. Yang

School of Medicine, University of Electronic Science and Technology of China, Chengdu, China

C. Yang $\cdot$ W. Le $\cdot$ Y. Xiang

Chinese Academy of Sciences Sichuan Translational Medicine Research Hospital, Chengdu 610072,

China heart. We proposed that CTRP9 could play multiple positive and beneficial roles in vascular lesions in ischemic stroke (IS). Here, we aimed to study the relationship between serum CTRP9 and the etiology, severity, and prognosis of IS patients.

Methods: A total of 302 patients with IS and 173 non-stroke controls were selected from the same hospital, and all patients with IS were followed up 12 months after stroke onset. Stroke etiology was classified according to the Trial of ORG 10172 in Acute Stroke Treatment classification. Symptomatic severity was determined using the National Institutes of Health Stroke Scale score. The lesion volume of acute cerebral ischemia was measured using magnetic resonance imaging (MRI). The unfavorable functional outcome was a combination of death or major disability 12 months after stroke onset.

\section{J. Xin · Z. Liu · F. Fan · F. Jin · Q. Wang}

Department of Neurology, General Hospital of

Western Theater Command, Chengdu, Sichuan,

China

Y. Li

Statistic Office, Department of Information, General Hospital of Western Theater Command, Chengdu, China

F. Guo $\cdot$ N. Yu $\cdot$ Y. Xiang

Department of Neurology, Sichuan Provincial

People's Hospital, University of Electronic Science and Technology of China, Chengdu, China 
Receiver operating characteristic (ROC) curves and integrated discrimination improvement (IDI) and net reclassification improvement (NRI) statistics were applied in the statistical analysis.

Results: We found that serum CTRP9 levels and the ratios of CTRP9/total cholesterol (TC), CTRP9/triglyceride (TG), CTRP9/low-density lipoprotein cholesterol (LDL-C), and CTRP9/ high-density lipoprotein cholesterol (HDL-C) were associated with the presence of IS. Moreover, the serum CTRP9 concentration was positively associated with the severity of IS. Incorporation of CTRP9/LDL-C levels into a fully adjusted model for IS-cardioembolic (CE) improved discrimination and calibration, and significantly improved reclassification. In addition, CTRP9 was a predictor of unfavorable functional outcomes.

Conclusions: All the findings indicated that serum CTRP9 could be a promising bloodderived biomarker for the early evaluation and prognosis assessment of IS.

Trial Registration: Chinese Clinical Trial Registry, ChiCTR1800020330.

Keywords: Ischemic stroke; Serum CTRP9; Cardioembolic; Prognosis

\section{Key Summary Points}

According to our previous review, we proposed that $\mathrm{C} 1 \mathrm{q}$ tumor necrosis factorrelated protein 9 (CTRP9) could play multiple roles in vascular lesions in ischemic stroke (IS).

The present study explored the association between serum CTRP9 levels and the presence of IS, and investigated the associations between CTRP9 concentrations and the etiology, severity, and prognosis of IS.

All the findings indicated that the serum CTRP9 could be a promising bloodderived biomarker for the early evaluation and prognosis assessment of IS.

\section{INTRODUCTION}

Globally, stroke is a serious public health problem and a leading cause of disability and mortality [1]. Specifically, China has 2.5 million new stroke cases each year and currently has 7.5 million stroke survivors [2]; approximately twothirds of all stroke cases are ischemic stroke (IS) [3]. Early evaluation of the etiology, severity, and prognosis of IS is crucial for the immediate application of appropriate therapy to patients [2]. Although the development of medical imaging has facilitated the early diagnosis of stroke, it is not widely used in the vast, underdeveloped areas in China and is not an option for patients with certain psychiatric conditions such as claustrophobia. Further, stroke is divided into different etiologies for which treatment is not the same. The appropriate treatment plan determines the prognosis of patients, so distinguishing stroke etiology as early as possible is key. Finally, early prediction of prognosis also plays a very important role in the rational arrangement of follow-up treatment. Therefore, the development of a fast and accurate evaluative and prognostic indicator of IS is crucial.

Identifying blood-derived biomarkers of stroke is an active field of research, since their potential use is not limited to diagnosis and differentiation, but can be applied to prognosis and patient monitoring, monitoring the effectiveness of applied therapy, and/or diagnosing possible complications. According to our previous review, C1q tumor necrosis factor (TNF)related protein 9 (CTRP9) plays a vital role in the cardiovascular disease process, including regulating energy metabolism, modulating vasomotion, protecting endothelial cells, inhibiting platelet activation, inhibiting pathological vascular remodeling, stabilizing atherosclerotic plaques, and protecting the heart [4]. Hence, we proposed that CTRP9 could play multiple positive and beneficial roles in IS.

In addition, in clinical practice, there are obvious individual differences in the absolute values of some blood-derived biomarkers, which limits their understanding and 
application by researchers and clinicians. Therefore, ratios or other calculations that use standard or other related clinical indicators to obtain a new indicator, such as prothrombin time (PT) and international normalized ratio (INR), could lead to better stability and comparability, which is convenient for research and clinical use [5]. The low-density lipoprotein cholesterol (LDL-C)/high-density lipoprotein cholesterol (HDL-C) ratio has also emerged as the best single lipid predictor of coronary artery disease (CAD) risk [6]. Therefore, the ratio of the level of CTRP9, as an adipocytokine, to blood lipid levels (such as CTRP9/total cholesterol [TC], CTRP9/triglyceride [TG], CTRP9/LDL-C, CTRP9/HDL-C) may be another stable evaluative and prognostic indicator of IS.

The association between CTRP9 and IS has not been elucidated thus far. Herein, in this study, we compared the serum CTRP9 concentrations between patients with IS and nonstroke controls, explored the association between serum CTRP9 levels and the presence of IS, and investigated the associations between CTRP9 concentrations and the etiology and severity of IS. To better understand and further extend knowledge on the predictive role of serum CTRP9 levels after IS, we prospectively investigated the relationship between serum CTRP9 levels and functional outcome in patients with IS.

\section{METHODS}

\section{Compliance with Ethics Guidelines}

This study was approved by the Ethical Review Board of the General Hospital of Western Theater Command (No.2018ky06). This study was conducted in compliance with the ethical standards of the responsible institution on human subjects and with the Helsinki Declaration. Participants included in the registry were asked to provide written informed consent for study participation.

\section{Study Design and Participant Selection}

Patients with IS were recruited from the Department of Neurology in this hospital between January 2018 and January 2020. The inclusion criteria were as follows [7]: (1) age $\geq 22$ years; (2) a diagnosis of IS as detected by magnetic resonance imaging (MRI) performed within $48 \mathrm{~h}$ of symptom onset; (3) ability and willingness of the patients or their direct family members to sign the informed consent form; and (4) admission time $>6 \mathrm{~h}$ from stroke onset. Participants were excluded if there was evidence of [8]: (1) recurrent stroke; (2) current pregnancy (for women); or (3) an inability to participate in the follow-up examinations. At admission, all patients underwent brain computed tomography (CT) scanning to rule out cerebral hemorrhage. None of the patients received thrombolysis, because they were admitted later than $6 \mathrm{~h}$ from symptom onset. Stroke etiology was classified according to the Trial of ORG 10172 in Acute Stroke Treatment (TOAST) classification [9].

Non-stroke controls without a history of cerebrovascular disease were selected during the same time period. The non-stroke participants were enrolled from the Health Physical Examination Center of the same hospital where they visited for annual health check-ups.

\section{Biochemical and Molecular Investigations}

Following a previously published protocol [10], fasting blood samples were collected between 06:00 a.m. and 07:00 a.m. within $24 \mathrm{~h}$ of admission to avoid variations related to possible circadian rhythm effects, and aliquots were made for routine blood tests, including white blood cell (WBC) count, C-reactive protein (CRP), fasting glucose, hemoglobin A1C (HbA1C), TC, TG, HDL-C, and LDL-C, in the Department of Clinical Laboratory. The serum was separated and frozen at $-80^{\circ} \mathrm{C}$ until CTRP9 measurement.

In accordance with previous studies [11, 12], we employed a commercial enzyme-linked immunosorbent assay (ELISA) kit (SER877Hu, USCN Life Science, China) to measure the 
serum CTRP9 concentration in all participants according to the manufacturer's instructions. Samples and standards were measured in duplicate, and the means of the duplicates were used for statistical analysis.

The minimum detectable dose of human CTRP9 was typically less than $6.32 \mathrm{pg} / \mathrm{mL}$. The intra- and inter-assay coefficients of variation were $<10 \%$ and $<12 \%$, respectively. The linear range of the assay was $15.6-1000 \mathrm{pg} / \mathrm{mL}$. The assay had high sensitivity and specificity for the detection of human CTRP9, and it was observed that there was no significant cross-reactivity or interference between human CTRP9 and other CTRPs or other analogs in human blood. Samples and standards were measured in duplicate, and the means of the duplicates were used for statistical analysis.

\section{Assessment of Stroke Symptomatic Severity}

All the patients were assessed by two welltrained neurologists, and the stroke symptomatic severity was recorded according to the National Institutes of Health Stroke Scale (NIHSS) [13], which rates stroke symptomatic severity on a scale of $0-42$, with a higher score indicating more severe impairment of the brain.

\section{Assessment of Acute Cerebral Ischemia}

All the patients with IS underwent brain MRI scanning (Achieva 3.0T, Philips, Netherlands) to determine the location and extent of the acute cerebral ischemia (ACI). The assessment of ACI was performed following a previous protocol [14]. To quantify the lesion extent, we measured the maximal transverse diameter of the lesion and a second diameter perpendicular to the first one on the same slice on diffusion-weighted imaging (DWI) of MRI. The craniocaudal extent was measured by multiplying the number of consecutive slices in which the lesion was present by the sum of the slice thickness $(6 \mathrm{~mm})$ and the intersection gap $(0.6 \mathrm{~mm})$. The lesion volume of ACI was then calculated by the ellipsoid formula:

$$
\text { Lesion volume }=4 / 3 \cdot \pi \cdot r 1 \cdot r 2 \cdot r 3 \text {, }
$$

where $r 1$ and $r 2$ correspond to half of the two transverse diameters, and $r 3$ corresponds to half of the craniocaudal extent. A blinded, second reading of the DW images was performed by two other experienced neuroradiologists to assess the validity of the measurements.

\section{Assessment of Other Clinical Variables}

Baseline data on demographic characteristics (age and sex), anthropometric measurements (systolic blood pressure [SBP], diastolic blood pressure [DBP], and body mass index [BMI]), lifestyle risk factors (cigarette smoking and alcohol consumption), and medical history (hypertension, atrial fibrillation [AF], diabetes mellitus, and other kinds of vascular diseases) were collected within $24 \mathrm{~h}$ of hospital admission.

\section{End Points and Follow-Up}

We followed the patients for a median of 12 months using a standard questionnaire, and telephone or household contact by physician investigators. An unfavorable functional outcome was defined as a combination of death and major disability (modified Rankin Scale score $[\mathrm{mRS}] \geq 3$ ) [15].

\section{Statistical Analysis}

The differences in baseline characteristics between the two groups were examined using the independent $t$-test, Mann-Whitney $U$ test, or chi-squared test as appropriate. The influence of serum CTRP9 levels on the presence and subtype of IS was determined by multivariate logistic regression analysis, which allowed adjustment for confounding factors. Cox proportional hazards regression models were also used to estimate the risk of functional outcomes when appropriate. Clinical variables with $P<0.05$ on univariate analysis, in addition to sex and age, were incorporated into multivariate logistic regression analyses and Cox proportional hazards regression models. The results 
were expressed as adjusted odds ratios (ORs) or hazard ratios (HRs) with the corresponding 95\% confidence intervals (CIs). The clinical value of adding CTRP9 to the existing risk factors for identifying or predicting the presence, subtype, and outcome of IS was calculated with receiver operating characteristic (ROC) curves, the integrated discrimination improvement (IDI) index, and the net reclassification improvement (NRI) index. Spearman correlation analysis was used to analyze the bivariate correlations between the serum CTRP9 concentration and volume and NIHSS scores. The statistical analysis was performed using IBM SPSS Statistics for Windows, version 16.0 (IBM Corp., Armonk, NY, USA), $\mathrm{R}$ programming language (version 2.15.1), and GraphPad Prism 5.0 software (GraphPad Software, La Jolla, CA, USA). A $P$ value $<0.05$ indicated significance.

\section{RESULTS}

\section{CTRP9 and IS}

A total of 351 patients with IS were screened, and 302 patients met the inclusion criteria and finished 12 months of follow-up. The baseline characteristics of all participants are shown in Table 1. The effects of serum CTRP9 concentration and the ratios of CTRP9/TC, CTRP9/TG, CTRP9/LDL-C, and CTRP9/HDL-C on the presence of IS were examined using multivariate logistic regression analysis. When sex, age, BMI, hypertension, diabetes mellitus, WBC, and CRP were adjusted, the serum CTRP9 concentration and the ratios of CTRP9/TC, CTRP9/TG, CTRP9/ LDL-C, and CTRP9/HDL-C were associated with the presence of IS (Table 2). Further, the addition of CTRP9 or ratios of CTRP9/TC, CTRP9/ TG, CTRP9/LDL-C, and CTRP9/HDL-C to a model adjusted for sex, age, BMI, hypertension, diabetes mellitus, and WBC led to a further improvement in model performance (Table 3). Spearman correlation analysis showed that the serum CTRP9 concentration was positively associated with the NIHSS score $(r=0.267$, $P<0.001)$ and lesion volume $(r=0.282$, $P<0.001)$ in patients with IS.

\section{CTRP9 and Cardioembolic (CE) IS}

We studied 64 patients with IS-CE and 238 patients with IS-non-CE. The baseline characteristics of the two groups are shown in Supplementary Table 1 . The independent effect of serum CTRP9 concentration on IS-CE was examined using binary logistic regression analysis. When sex, age, AF, hypertension, hyperlipidemia, volume, CRP, and NIHSS score were adjusted, the serum CTRP9 concentration and the ratios of CTRP9/TC, CTRP9/TG, CTRP9/ LDL-C, and CTRP9/HDL-C were associated with IS-CE (Table 4). Meanwhile, when sex, age, hypertension, hyperlipidemia, volume, CRP, and NIHSS score were adjusted, the serum CTRP9 concentration and the ratios of CTRP9/ TC, CTRP9/TG, CTRP9/LDL-C, and CTRP9/ HDL-C were associated with IS-CE (Table 4). Further, only the addition of CTRP9/LDL-C to a model adjusted for sex, age, AF, and hypertension led to a further improvement in model performance [increase in AUC from 0.883 for sex, age, AF, hypertension to 0.908 for sex, age, $\mathrm{AF}$, hypertension plus CTRP9/LDL-C, $P=0.046$; IDI $=0.069, P<0.001 ; \quad$ NRI $=0.466, P<0.001]$ (Table 5).

\section{Association Between Serum CTRP9 Level and the Unfavorable Functional Outcome of IS}

Referring to other research [16], we divided these patients into tertiles according to CTRP9 level: patients with CTRP9 in the lowest tertile (1st tertile, CTRP9 $\leq 71 \mathrm{ng} / \mathrm{mL}, n=100$ ); those with CTRP9 in the middle tertile (2nd tertile, CTRP9 71-104 ng/mL, $n=102$ ); and those with CTRP9 in the highest tertile (3rd tertile, CTRP9 $>104 \mathrm{ng} / \mathrm{mL}, \quad n=100)$. The baseline characteristics of the three groups are shown in Supplementary Table 2. A total of 67 participants $(22.19 \%)$ experienced unfavorable functional outcomes. After adjustment for sex, age, atrial fibrillation, volume, WBC, fasting glucose, and NIHSS score, the HR for CTRP9 > $104 \mathrm{ng} /$ $\mathrm{mL}$ was 3.707 for unfavorable functional outcome (Table 6). In addition, only the addition of CTRP9 to a model adjusting for sex, age, and 
Table 1 Demographics and clinical characteristics of all participants

\begin{tabular}{|c|c|c|c|}
\hline Variable & IS $(n=302)$ & Control $(n=173)$ & $P$ value \\
\hline Age, years & $65(54-73)$ & $63(51-71)$ & 0.064 \\
\hline Male & $191(63.25)$ & $103(59.54)$ & 0.423 \\
\hline BMI, $\mathrm{kg} / \mathrm{m}^{2}$ & $24.43(22.60-26.16)$ & $22.67(20.92-24.92)$ & $<0.001$ \\
\hline Cigarette smoking & $144(47.68)$ & $78(45.09)$ & 0.585 \\
\hline Alcohol consumption & $120(39.74)$ & $56(32.37)$ & 0.110 \\
\hline Hypertension & 199 (65.89) & $70(40.46)$ & $<0.001$ \\
\hline Diabetes mellitus & $100(33.11)$ & $24(13.87)$ & $<0.001$ \\
\hline Hyperlipidemia & $66(21.85)$ & $39(22.54)$ & 0.862 \\
\hline $\mathrm{SBP}(\mathrm{mmHg})$ & $145(132-159.25)$ & $130(119.5-145)$ & $<0.001$ \\
\hline DBP (mmHg) & $88(78-96)$ & $80(70-86)$ & $<0.001$ \\
\hline WBC $\left(\times 10^{9} / \mathrm{L}\right)$ & $7.05(5.69-9.18)$ & $5.70(4.72-6.87)$ & $<0.001$ \\
\hline $\mathrm{CRP}(\mathrm{mg} / \mathrm{L})$ & $2.64(1.09-8.63)$ & $1.01(0.54-2.40)$ & $<0.001$ \\
\hline Fasting glucose $(\mathrm{mmol} / \mathrm{L})$ & $5.66(4.98-7.49)$ & $5.08(4.64-5.81)$ & $<0.001$ \\
\hline HbAlc (\%) & $6.00(5.50-6.82)$ & $5.4(4.9-5.9)$ & $<0.001$ \\
\hline $\mathrm{TC}(\mathrm{mmol} / \mathrm{L})$ & $4.71(3.85-5.63)$ & $4.72(4.04-5.59)$ & 0.876 \\
\hline $\mathrm{TG}(\mathrm{mmol} / \mathrm{L})$ & $1.28(0.95-1.84)$ & $1.36(0.94-1.84)$ & 0.698 \\
\hline $\mathrm{HDL}-\mathrm{C}(\mathrm{mmol} / \mathrm{L})$ & $1.29(1.11-1.61)$ & $1.35(1.13-1.63)$ & 0.490 \\
\hline LDL-C $(\mathrm{mmol} / \mathrm{L})$ & $2.75(2.15-3.56)$ & $2.81(2.40-3.38)$ & 0.677 \\
\hline CTRP9 (ng/mL) & $86.92(62.14-117.96)$ & $60.17(49.33-75.06)$ & $<0.001$ \\
\hline CTRP9/TC & $16.94(12.88-24.31)$ & $12.57(10.44-15.38)$ & $<0.001$ \\
\hline CTRP9/TG & $63.58(40.19-106.01)$ & $43.53(32.58-67.03)$ & $<0.001$ \\
\hline CTRP9/LDL-C & $28.12(21.49-44.68)$ & $20.95(17.06-26.77)$ & $<0.001$ \\
\hline CTRP9/HDL-C & $64.62(45.13-94.29)$ & $46.37(35.74-54.12)$ & $<0.001$ \\
\hline \multicolumn{4}{|l|}{ TOAST } \\
\hline LAA & $174(57.62)$ & & \\
\hline $\mathrm{CE}$ & $64(21.20)$ & & \\
\hline SVD & $54(17.88)$ & & \\
\hline SOE & $3(0.10)$ & & \\
\hline SUE & $7(2.32)$ & & \\
\hline NIHSS & $6.5(4,10)$ & & \\
\hline $\mathrm{mRS}$ & $1(0,2)$ & & \\
\hline
\end{tabular}


Table 1 continued

\begin{tabular}{llll}
\hline Variable & IS $(\boldsymbol{n}=\mathbf{3 0 2})$ & Control $(\boldsymbol{n}=\mathbf{1 7 3})$ & $\boldsymbol{P}$ value \\
\hline $\mathrm{mRS} \geq 3$ & $64(21.19)$ & & \\
\hline
\end{tabular}

Values are given as the median (IQR) or as a number $(n)$ with the percentage in parentheses

$I S$ ischemic stroke, $B M I$ body mass index, $S B P$ systolic blood pressure, $D B P$ diastolic blood pressure, $W B C$ white blood cell, $C R P$ C-reactive protein, $H b A 1 c$ hemoglobin A1c, $T C$ total cholesterol, $T G$ triglyceride, $H D L-C$ high-density lipoprotein cholesterol, $L D L-C$ low-density lipoprotein cholesterol, CTRP9 C1q TNF-related protein 9, TOAST Trial of ORG 10172 in Acute Stroke Treatment, $L A A$ large artery atherosclerosis, $C E$ cardioembolic, $S V D$ small vessel disease, SOE other determined etiology, $S U E$ undetermined etiology

NIHSS score led to a further improvement in model performance [increase in AUC from 0.811 for sex, age, and NIHSS score to 0.846 for sex, age, NIHSS score plus CTRP9, $P=0.045$; IDI $=0.081, P<0.001 ; \quad \mathrm{NRI}=0.617, P<0.001]$ (Table 7).

\section{DISCUSSION}

CTRP9, a novel member of the C1q/TNF superfamily, was identified in 2009 [17, 18]. IS and $\mathrm{CAD}$ share considerable pathogenesis, from risk factors to vascular lesions followed by different target organ damage [19-21]. According to our previous review, CTRP9 could play multiple positive roles in vascular lesions in IS [4]. In addition, the ratios of blood lipid indicators to each other may likely affect the progression of atherosclerosis [22], and the LDL-C/HDL-C ratio has already been found to be superior to single lipid predictors of cardiovascular disease risk [22]. The ratios of CTRP9 to lipids (ratios of CTRP9/TC, CTRP9/TG, CTRP9/LDL-C, CTRP9/ HDL-C) may be a better evaluative and prognostic indicator of IS.

In this study, we found that the serum CTRP9 concentration and ratios of CTRP9/TC, CTRP9/TG, CTRP9/LDL-C, and CTRP9/HDL-C were different between stroke patients and nonstroke controls, and the serum CTRP9 concentration and the ratios of CTRP9/TC, CTRP9/TG, CTRP9/LDL-C, and CTRP9/HDL-C in IS were significantly higher in stroke patients than in non-stroke controls. CTRP9 and the ratios of
CTRP9/TC, CTRP9/TG, CTRP9/LDL-C, and CTRP9/HDL-C could play negative roles in patients with IS.

However, these findings were inconsistent with our previous proposal [4]. Two possible explanations are as follows. First, CTRP9 is generated in the process of atherosclerosis. Conflicting results regarding the correlation between CTRP9 and metabolic factors $[11,23,24]$ and atherosclerotic lesions [25-27] have been reported, indicating that CTRP9 might be more than just a purely protective factor. Second, the CTRP9 concentration might change dynamically. In the early stage of disease development, CTRP9 may show protective effects: when the disease occurs in the acute stage, the level of CTRP9 may be increased to enhance its anti-inflammatory and other protective effects. In the later stage of the disease, the level of CTRP9 may decrease gradually with the improvement of the disease. These may indicate that CTRP9 is a better predictor of disease progression.

In the study, the time point we detected did not reflect the CTRP9 level at the ultra-early stage of IS $(<6 \mathrm{~h}$ from stroke onset). The reasons are as follows. According to the existing guidelines for the diagnosis and treatment of acute stroke, the hyperacute stage is within $6 \mathrm{~h}$ after the onset of stroke, during which patients may receive intravenous thrombolysis or (and) intra-arterial thrombolysis according to their actual situation. We did not include these specific treatments in this study, considering 
Table 2 Independent factors associated with IS in binary logistic regression models

\begin{tabular}{|c|c|c|c|}
\hline & Variable & OR (95\% CI) & $P$ value \\
\hline \multirow[t]{3}{*}{ Model 1} & Hypertension & $2.682(1.652-4.355)$ & $<0.001$ \\
\hline & WBC & $1.343(1.184-1.523)$ & $<0.001$ \\
\hline & CTRP9 & $1.031(1.022-1.041)$ & $<0.001$ \\
\hline \multirow[t]{4}{*}{ Model 2} & BMI & $1.127(1.039-1.223)$ & 0.004 \\
\hline & Hypertension & $2.376(1.486-3.801)$ & $<0.001$ \\
\hline & WBC & $1.313(1.163-1.481)$ & $<0.001$ \\
\hline & CTRP9/TC & $1.120(1.078-1.162)$ & $<0.001$ \\
\hline \multirow[t]{4}{*}{ Model 3} & BMI & $1.151(1.063-1.247)$ & 0.001 \\
\hline & Hypertension & $2.449(1.544-3.886)$ & $<0.001$ \\
\hline & Diabetes mellitus & $2.138(1.204-3.798)$ & 0.101 \\
\hline & CTRP9/TG & $1.017(1.011-1.024)$ & $<0.001$ \\
\hline \multirow[t]{4}{*}{ Model 4} & BMI & $1.150(1.058-1.250)$ & 0.001 \\
\hline & Hypertension & $2.359(1.476-3.771)$ & $<0.001$ \\
\hline & Diabetes mellitus & $1.900(1.066-3.385)$ & 0.030 \\
\hline & CTRP9/LDL-C & $1.063(1.041-1.084)$ & $<0.001$ \\
\hline \multirow[t]{4}{*}{ Model 5} & BMI & $1.111(1.024-1.204)$ & 0.011 \\
\hline & Hypertension & $2.345(1.473-3.735)$ & $<0.001$ \\
\hline & WBC & $1.316(1.167-1.484)$ & $<0.001$ \\
\hline & CTRP9/HDL-C & $1.026(1.017-1.036)$ & $<0.001$ \\
\hline
\end{tabular}

Model 1: sex, age, BMI, hypertension, diabetes mellitus, WBC, CRP, CTRP9. Model 2: sex, age, BMI, hypertension, diabetes mellitus, WBC, CRP, CTRP9/TC. Model 3: sex, age, BMI, hypertension, diabetes mellitus, WBC, CRP, CTRP9/ TG. Model 4: sex, age, BMI, hypertension, diabetes mellitus, WBC, CRP, CTRP9/LDL-C. Model 5: sex, age, BMI, hypertension, diabetes mellitus, WBC, CRP, CTRP9/HDL-C

$B M I$ body mass index, $W B C$ white blood cell, $C R P$ C-reactive protein, $T C$ total cholesterol, $T G$ triglyceride, $H D L-C$ highdensity lipoprotein cholesterol, $L D L-C$ low-density lipoprotein cholesterol, CTRP9 C1q/TNF-related protein $9, O R$ odds ratio, $C I$ confidence interval

their impact on disease characteristics and prognosis.

It may also be that the increase in CTRP9 levels induced by vascular lesions reflects the severity of the lesions in the body. In this research, we found that the serum CTRP9 concentration was positively associated with the symptomatic severity of IS, reflected by the NIHSS score, and the degree of ACI, reflected by the lesion volume measured by MRI-DWI. MRI-
DWI is particularly useful for ACI evaluation during the first $6 \mathrm{~h}$ from IS onset [28]. In this study, we ensured that the lesion volume data reflected the degree of ACI for all the patients undergoing brain MRI-DWI $48 \mathrm{~h}$ after stroke onset. In addition, we found that the serum CTRP9 concentration and ratios of CTRP9/TC, CTRP9/TG, CTRP9/LDL-C, and CTRP9/HDL-C significantly improved the AUC. The serum CTRP9 concentration and ratios of CTRP9/TC, 
Table 3 Reclassification and discrimination statistics for the presence of IS by CTRP9 and the ratios of CTRP9/TC, CTRP9/TG, CTRP9/LDL-C, and CTRP9/HDL-C

\begin{tabular}{|c|c|c|c|c|c|c|}
\hline & \multicolumn{2}{|l|}{ ROC } & \multicolumn{2}{|l|}{ NRI (continuous) } & \multicolumn{2}{|l|}{ IDI } \\
\hline & $\overline{\text { AUC (95\% CI) }}$ & $P$ value & Value (95\% CI) & $P$ value & $\overline{\text { Value (95\% CI) }}$ & $P$ value \\
\hline Model 1 & $0.771(0.730-0.808)$ & - & $1.0[\mathrm{Ref}]$ & - & $1.0[\mathrm{Ref}]$ & - \\
\hline Model 2 & $0.836(0.799-0.868)$ & $<0.001$ & $0.826(0.661-0.990)$ & $<0.001$ & $0.120(0.094-0.147)$ & $<0.001$ \\
\hline Model 3 & $0.823(0.786-0.857)$ & $<0.001$ & $0.630(0.462-0.799)$ & $<0.001$ & $0.096(0.071-0.200)$ & $<0.001$ \\
\hline Model 4 & $0.811(0.773-0.845)$ & 0.001 & $0.437(0.260-0.613)$ & $<0.001$ & $0.073(0.050-0.096)$ & $<0.001$ \\
\hline Model 5 & $0.825(0.788-0.858)$ & $<0.001$ & $0.604(0.435-0.773)$ & $<0.001$ & $0.097(0.073-0.122)$ & $<0.001$ \\
\hline Model 6 & $0.817(0.780-0.851)$ & $<0.001$ & $0.749(0.585-0.914)$ & $<0.001$ & $0.08(0.057-0.103)$ & $<0.001$ \\
\hline
\end{tabular}

Model 1: sex, age, BMI, hypertension, diabetes mellitus, WBC. Model 2: Model 1+CTRP9. Model 3: Model 1+CTRP9/TC. Model 4: Model 1+CTRP9/TG. Model 5: Model 1+CTRP9/LDL-C. Model 6: Model $1+$ CTRP9/HDL-C

$B M I$ body mass index, $W B C$ white blood cell, $T C$ total cholesterol, $T G$ triglyceride, $H D L-C$ high-density lipoprotein cholesterol, $L D L-C$ low-density lipoprotein cholesterol, CTRP9 C1q/TNF-related protein 9, AUC area under the curve, $N R I$ net reclassification improvement, $I D I$ integrated discrimination improvement

CTRP9/TG, CTRP9/LDL-C, and CTRP9/HDL-C significantly improved diagnostic efficacy over the clinical models alone as measured by the NRI and IDI. All the findings indicated that the serum CTRP9 concentration and ratios of CTRP9/TC, CTRP9/TG, CTRP9/LDL-C, and CTRP9/HDL-C could be promising bloodderived biomarkers for the early evaluation of IS, especially CTRP9/TC.

There are many etiologies of IS, and treatments differ for different subtypes. The TOAST criteria for the subtypes of IS include CE stroke, large artery atherosclerosis (LAA), small vessel disease (SVD), other determined etiology (SOE), and undetermined etiology (SUE). CE events are the most common cause of IS [29, 30]. Due to the similarities in the clinical presentation [31] and the lack of biomarkers, it may be difficult to distinguish between etiologies, leading to delayed diagnosis and inappropriate treatment [32]. Biomarkers could serve as objective measurements that are indicators of normal biological or pathogenic processes. The use of blood biomarkers has been suggested as a promising tool to improve stroke etiology classification [33]. For example, some promising candidates, such as natriuretic peptides, have been associated with IS-CE etiology [34], while others, such as lipoprotein-associated phospholipase A2, have been related to IS-LAA etiology [35]. Several studies have also pointed out the usefulness of assessing gene expression in blood to discriminate between IS etiologies [36]; however, no biomarker has yet been approved for use in clinical practice.

Given that the most common etiologies of cardioembolic stroke are atrial fibrillation, valvular heart disease, or dilated cardiomyopathy, which is different from IS-non-CE [37, 38], it is possible that the serum CTRP9 concentration differentiates CE from IS-non-CE. In this study, we first found that the serum CTRP9 concentration and the ratios of CTRP9/TC, CTRP9/TG, CTRP9/LDL-C, and CTRP9/HDL-C were significantly different between IS-CE and IS-non-CE patients. Remarkably, the serum CTRP9 concentration and the ratios of CTRP9/ TC, CTRP9/TG, CTRP9/LDL-C, and CTRP9/ HDL-C were significantly increased in patients with IS-CE compared to patients with IS-nonCE. Two possible explanations are as follows. First, IS-CE usually leads to acute injury of large vessels or multiple vessels, and since CTRP9 may play an important role in vascular injury, the body may increase the serum CTRP9 concentration in reactive acute tissue injury. 
Table 4 Independent factors associated with IS-CE in binary logistic regression models

\begin{tabular}{|c|c|c|c|c|c|}
\hline & \multirow[t]{2}{*}{ Variable } & \multicolumn{2}{|l|}{ Model with AF } & \multicolumn{2}{|l|}{ Model without AF } \\
\hline & & OR (95\% CI) & $P$ value & OR (95\%CI) & $P$ value \\
\hline \multirow[t]{3}{*}{ Model 1} & $\mathrm{AF}$ & $36.713(16.221-83.095)$ & $<0.001$ & - & - \\
\hline & Hypertension & $0.328(0.149-0.726)$ & 0.006 & $0.279(0.145-0.538)$ & $<0.001$ \\
\hline & CTRP9 & $1.014(1.006-1.022)$ & 0.001 & $1.013(1.006-1.019)$ & $<0.001$ \\
\hline \multirow[t]{3}{*}{ Model 2} & $\mathrm{AF}$ & $34.264(14.951-78.525)$ & $<0.001$ & - & - \\
\hline & Hypertension & $0.343(0.153-0.771)$ & 0.010 & $0.292(0.149-0.571)$ & $<0.001$ \\
\hline & CTRP9/TC & $1.070(1.036-1.105)$ & $<0.001$ & $1.070(1.042-1.097)$ & $<0.001$ \\
\hline \multirow[t]{3}{*}{ Model 3} & $\mathrm{AF}$ & $31.333(14.373-68.308)$ & $<0.001$ & - & - \\
\hline & Hypertension & $0.335(0.153-0.732)$ & 0.006 & $0.345(0.185-0.641)$ & 0.001 \\
\hline & CTRP9/TG & $1.006(1.001-1.010)$ & 0.013 & $1.007(1.003-1.011)$ & $<0.001$ \\
\hline \multirow[t]{3}{*}{ Model 4} & $\mathrm{AF}$ & $32.209(14.182-73.151)$ & $<0.001$ & - & - \\
\hline & Hypertension & $0.349(0.155-0.786)$ & 0.011 & $0.351(0.185-0.666)$ & 0.001 \\
\hline & CTRP9/LDL-C & $1.036(1.019-1.054)$ & $<0.001$ & $1.039(1.024-1.053)$ & $<0.001$ \\
\hline \multirow[t]{3}{*}{ Model 5} & $\mathrm{AF}$ & $38.066(16.710-86.716)$ & $<0.001$ & - & - \\
\hline & Hypertension & $0.303(0.136-0.672)$ & 0.003 & $0.268(0.140-0.512)$ & $<0.001$ \\
\hline & CTRP9/HDL-C & $1.015(1.007-1.023)$ & $<0.001$ & $1.014(1.007-1.020)$ & $<0.001$ \\
\hline
\end{tabular}

Model 1: sex, age, AF, hypertension, hyperlipidemia, volume, CRP, NIHSS, CTRP9. Model 2: sex, age, AF, hypertension, hyperlipidemia, volume, CRP, NIHSS, CTRP9/TC. Model 3: sex, age, AF, hypertension, hyperlipidemia, volume, CRP, NIHSS, CTRP9/TG. Model 4: sex, age, AF, hypertension, hyperlipidemia, volume, CRP, NIHSS, CTRP9/LDL-C. Model 5: sex, age, AF, hypertension, hyperlipidemia, volume, CRP, NIHSS, CTRP9/HDL-C

$C E$ cardioembolic, $B M I$ body mass index, $A F$ atrial fibrillation, $C R P$ C-reactive protein, $T C$ total cholesterol, $T G$ triglyceride, $H D L-C$ high-density lipoprotein cholesterol, $L D L-C$ low-density lipoprotein cholesterol, $C T R P 9 \mathrm{C} 1 \mathrm{q} / \mathrm{TNF}$ related protein $9, O R$ odds ratio, $C I$ confidence interval

Second, CTRP9 can be detected in heart tissue, and previous studies have also shown a potential role of CTRP9 in heart damage. Therefore, changes in the heart, including abnormal heart rhythms or structural changes, may cause rapid changes in serum CTRP9 levels. In addition, the addition of CTRP9/LDL-C significantly improved the resolution ability from IS-non-CE to IS-CE, as evidenced by the AUC, NRI, and IDI. It is reasonable to assume that the CTRP9/LDL$\mathrm{C}$ ratio may be an indicator that differentiates between IS-CE and IS-non-CE.

Predicting the functional prognosis among patients with IS during the acute phase may be useful in helping to select therapeutic strategies.
Biomarkers show potential predictive value, and several biomarkers have been studied as diagnostic, risk stratification, and prognostic tools, namely, S100 calcium binding protein B, C-reactive protein, matrix metalloproteinases, and cerebral natriuretic peptide. However, due to the low specificity of the related indicators and the inconsistency of baseline values obtained by various laboratories, they have not been widely used. Additional studies are necessary to identify reliable predictive markers for functional prognosis after IS [39]. Notably, most studies looked only at prognosis three months after stroke, with little attention given to longer-term outcomes. 
Table 5 Reclassification and discrimination statistics for presence of IS-CE by CTRP9 and the ratios of CTRP9/TC, CTRP9/TG, CTRP9/LDL-C, and CTRP9/HDL-C

\begin{tabular}{|c|c|c|c|c|c|c|}
\hline & \multicolumn{2}{|l|}{ ROC } & \multicolumn{2}{|l|}{ NRI (continuous) } & \multicolumn{2}{|l|}{ IDI } \\
\hline & $\overline{\text { AUC (95\%) }}$ & $P$ value & Value (95\%) & $P$ value & $\overline{\text { Value (95\%) }}$ & $P$ value \\
\hline Model 1 & $0.883(0.841-0.917)$ & - & $1.0[\mathrm{Ref}]$ & - & $1.0[\mathrm{Ref}]$ & - \\
\hline Model 2 & $0.899(0.859-0.930)$ & 0.213 & $0.449(0.179-0.719)$ & $<0.001$ & $0.0437(0.012$ to 0.076$)$ & 0.008 \\
\hline Model 3 & $0.905(0.866-0.935)$ & 0.103 & $0.594(0.326-0.862)$ & $<0.001$ & $0.070(0.030$ to 0.110$)$ & $<0.001$ \\
\hline Model 4 & $0.899(0.859-0.931)$ & 0.066 & $0.350(0.082-0.617)$ & 0.011 & $0.017(-0.005$ to 0.039$)$ & 0.124 \\
\hline Model 5 & $0.908(0.869-0.938)$ & 0.046 & $0.466(0.196-0.736)$ & $<0.001$ & $0.069(0.030$ to 0.109$)$ & $<0.001$ \\
\hline Model 6 & $0.897(0.857-0.929)$ & 0.253 & $0.596(0.329-0.864)$ & $<0.001$ & $0.049(0.017$ to 0.081$)$ & 0.003 \\
\hline
\end{tabular}

Model 1: sex, age, AF, hypertension. Model 2: Model $1+$ CTRP9. Model 2: Model $1+$ CTRP9/TC. Model 4: Model $1+$ CTRP9/TG. Model 5: Model 1 + CTRP9/LDL-C. Model 6: Model 1 + CTRP9/HDL-C

$A F$ atrial fibrillation, $T C$ total cholesterol, $T G$ triglyceride, $H D L-C$ high-density lipoprotein cholesterol, $L D L$ - $C$ low-density lipoprotein cholesterol, CTRP9 Clq/TNF-related protein 9, AUC area under the curve, NRI net reclassification improvement, IDI integrated discrimination improvement

Table 6 Independent factors associated with unfavorable outcome of IS in Cox regression models

\begin{tabular}{lccccc}
\hline & \multicolumn{2}{c}{ Unadjusted } & & & Adjusted $^{\mathbf{a}}$ \\
\cline { 2 - 3 } & \multicolumn{1}{r}{ P value } & HR $(\mathbf{9 5 \%}$ CI $)$ & & P value & HR (95\% CI) \\
\hline Sex & 0.548 & $0.861(0.528-1.403)$ & & 0.245 & $1.373(0.805-2.341)$ \\
Age & 0.011 & $1.027(1.006-1.047)$ & & 0.035 & $1.023(1.002-1.045)$ \\
AF & 0.008 & $1.958(1.188-3.226)$ & & 0.964 & $0.986(0.539-1.804)$ \\
Volume & $<0.01$ & $1.007(1.004-1.010)$ & & 0.504 & $1.001(0.997-1.006)$ \\
WBC & $<0.01$ & $1.135(1.060-1.216)$ & & 0.200 & $1.057(0.971-1.152)$ \\
Fasting glucose & 0.006 & $1.095(1.027-1.167)$ & & 0.111 & $1.063(0.986-1.145)$ \\
NIHSS & $<0.01$ & $1.112(1.079-1.147)$ & & $<0.01$ & $1.103(1.055-1.153)$ \\
CTRP9 tertiles & $<0.01$ & & & 0.002 & \\
CTRP9 2nd tertile & 0.052 & $2.179(0.992-4.785)$ & & 0.051 & $2.254(0.998-5.092)$ \\
CTRP9 3rd tertile & $<0.01$ & $4.222(2.042-8.832)$ & & 0.001 & $3.707(1.746-7.869)$ \\
\hline
\end{tabular}

$A F$ atrial fibrillation, $W B C$ white blood cell, CTRP9 C1q TNF-related protein 9, $H R$ hazard ratio, $C I$ confidence interval, CTRP9 2nd tertile CTRP9:71-104 ng/mL, CTRP9 3rd tertile CTRP9 > $104 \mathrm{ng} / \mathrm{mL}$

${ }^{a}$ Adjusted for sex, age, AF, volume, WBC, fasting glucose, NIHSS, CTRP9 tertiles

Based on other studies and our study, we found that serum CTRP9 controls atherosclerosis and is associated with symptomatic severity and lesion volume in ischemic stroke, which are in turn associated with the secondary consequences of stroke. Therefore, in the prospective study section, we documented that the event rate of unfavorable functional outcomes was higher among patients with high serum CTRP9 concentrations than among those 
Table 7 Reclassification and discrimination statistics for unfavorable outcome by CTRP9 and the ratios of CTRP9/TC, CTRP9/TG, CTRP9/LDL-C, and CTRP9/HDL-C

\begin{tabular}{|c|c|c|c|c|c|c|}
\hline & \multicolumn{2}{|l|}{ ROC } & \multicolumn{2}{|l|}{ NRI (continuous) } & \multicolumn{2}{|l|}{ IDI } \\
\hline & $\overline{\text { AUC (95\%) }}$ & $P$ value & Value (95\%) & $P$ value & Value (95\%) & $P$ value \\
\hline Model 1 & $0.811(0.762-0.853)$ & - & $1.0[\mathrm{Ref}]$ & - & $1.0[\mathrm{Ref}]$ & - \\
\hline Model 2 & $0.846(0.801-0.885)$ & 0.045 & 0.617 (0.355 to 0.880$)$ & $<0.001$ & 0.081 (0.035 to 0.126$)$ & $<0.001$ \\
\hline Model 3 & $0.829(0.781-0.869)$ & 0.205 & 0.374 (0.111 to 0.638$)$ & 0.005 & 0.041 (0.008 to 0.074$)$ & 0.014 \\
\hline Model 4 & $0.817(0.768-0.859)$ & 0.488 & $0.233(-0.029$ to 9.495$)$ & 0.081 & $0.019(-0.002$ to 0.03$)$ & 0.069 \\
\hline Model 5 & $0.820(0.772-0.862)$ & 0.385 & 0.267 (0.003 to 0.532$)$ & 0.048 & $0.025(-8 \mathrm{e}-04$ to 0.05$)$ & 0.058 \\
\hline Model 6 & $0.846(0.800-0.884)$ & 0.057 & $0.609(0.347$ to 0.870$)$ & $<0.001$ & $0.076(0.032$ to 0.120$)$ & $<0.001$ \\
\hline
\end{tabular}

Model 1: sex, age, NIHSS. Model 2: Model 1+CTRP9. Model 2: Model 1+CTRP9/TC. Model 4: Model $1+$ CTRP9/TG. Model 5: Model $1+$ CTRP9/LDL-C. Model 6: Model 1 + CTRP9/HDL-C

$T C$ total cholesterol, TG triglyceride, $H D L-C$ high-density lipoprotein cholesterol, $L D L-C$ low-density lipoprotein cholesterol, CTRP9 C1q/TNF-related protein 9, AUC area under the curve, NRI net reclassification improvement, IDI integrated discrimination improvement

with low serum CTRP9 concentrations. Further, high serum CTRP9 levels were associated with an increased risk of major disability and death. Inclusion of serum CTRP9 levels among the validated prognostic factors, including age, sex, and the NIHSS score, significantly improved the reclassification of risk of adverse clinical outcomes, as evidenced by the AUC, NRI, and IDI. These findings indicated that serum CTRP9 levels could be a promising blood-derived biomarker for prognosis assessment of IS.

Several limitations of this study need to be pointed out. First, as a pilot study, the sample sizes were too small, and all participants were from a single hospital, which might not represent the distribution of IS in the Chinese population. In particular, the sample size of the non-stroke control group was smaller than that of the IS group (not up to the usual 1:1 ratio), which may to some extent affect our results as well as the interpretation of the results. Second, we did not dynamically monitor the changes in CTRP9 levels.

\section{CONCLUSIONS}

Taken together, these findings indicated that the serum CTRP9 concentration and ratios of
CTRP9 to lipids could be promising bloodderived early evaluative biomarkers and a useful tool to predict prognosis in patients with IS at admission. Further studies are needed to determine the reliability and validity of CTRP9 in clinical practice and reveal the roles of CTRP9 in the pathogenesis of IS in cell or animal models.

\section{ACKNOWLEDGEMENTS}

We thank all subjects who participated in the study.

Funding. This study was supported in part by National Natural Science Foundation of China (NSFC) Fund (No. 81601112), Sichuan Department of Science and Technology Fund (No. 2018SZ0141), Research Project of Sichuan Health and Family Planning Commission (No. 16PJ014), China's Post-doctoral Science Fund (No. 2017M623357), Sichuan Science and Technology Program (No. 2020YFS0436), and Sichuan Medical Association Fund (No. Q17008). The study and the Rapid Service Fee were funded by above-mentioned funding. 
Authorship. All named authors meet the International Committee of Medical Journal Editors (ICMJE) criteria for authorship for this article, take responsibility for the integrity of the work as a whole, and have given their approval for this version to be published. We thank the study participants and their relatives and the clinical staff at all participating hospitals for their support and contribution to this project.

Authors' Contributions. All authors contributed to the study conception and design. Material preparation, data collection and analysis were performed by Cui Yang, Jia-yan Xin, Zhi-lan Liu, Fan Fan, Yun-ming Li, Fan Jin, Qing-song Wang, Fu-qiang Guo, Neng-wei Yu, Wei-dong Le, MD, Yang Xiang. The first draft of the manuscript was written by Cui Yang and all authors commented on previous versions of the manuscript. All authors read and approved the final manuscript.

Disclosures. Cui Yang, Jia-yan Xin, Zhi-lan Liu, Fan Fan, Yun-ming Li, Fan Jin, Qing-song Wang, Fu-qiang Guo, Neng-wei Yu, Wei-dong Le, Yang Xiang have nothing to disclose.

Compliance with Ethics Guidelines. This study was approved by the Ethical Review Board of the General Hospital of Western Theater Command (No.2018ky06). This study was conducted in compliance with the ethical standards of the responsible institution on human subjects as well as with the Helsinki Declaration. Participants included in the registry were asked to provide written informed consent for study participation.

Data Availability. The datasets generated during and analyzed during the current study are available from the corresponding author on reasonable request.

Open Access. This article is licensed under a Creative Commons Attribution-NonCommercial 4.0 International License, which permits any non-commercial use, sharing, adaptation, distribution and reproduction in any medium or format, as long as you give appropriate credit to the original author(s) and the source, provide a link to the Creative Commons licence, and indicate if changes were made. The images or other third party material in this article are included in the article's Creative Commons licence, unless indicated otherwise in a credit line to the material. If material is not included in the article's Creative Commons licence and your intended use is not permitted by statutory regulation or exceeds the permitted use, you will need to obtain permission directly from the copyright holder. To view a copy of this licence, visit http://creativecommons.org/licenses/bync/4.0/.

\section{REFERENCES}

1. Collaborators GBDS. Global, regional, and national burden of stroke, 1990-2016: a systematic analysis for the Global Burden of Disease Study 2016. Lancet Neurol. 2019;18:439-58.

2. Liu L, Wang D, Wong KS, Wang Y. Stroke and stroke care in China: huge burden, significant workload, and a national priority. Stroke. 2011;42: 3651-4.

3. Pillai AA, Tadi P, Kanmanthareddy A. Cardioembolic stroke. Treasure Island: StatPearls; 2021.

4. Yang C, Fan F, Sawmiller D, et al. C1q/TNF-related protein 9: A novel therapeutic target in ischemic stroke? J Neurosci Res. 2019;97:128-36.

5. Deitcher SR. Interpretation of the international normalised ratio in patients with liver disease. Lancet. 2002;359:47-8.

6. Goedeke L, Rotllan N, Canfran-Duque A, et al. MicroRNA-148a regulates LDL receptor and ABCA1 expression to control circulating lipoprotein levels. Nat Med. 2015;21:1280-9.

7. Zhu Z, Xu T, Guo D, et al. Serum hepatocyte growth factor is probably associated with 3-month prognosis of acute ischemic stroke. Stroke. 2018;49: 377-83.

8. Peng $H$, Zhu F, Shi J, et al. Serum soluble corin is decreased in stroke. Stroke. 2015;46:1758-63.

9. Adams HP Jr, Bendixen BH, Kappelle LJ, et al. Classification of subtype of acute ischemic stroke. Definitions for use in a multicenter clinical trial. 
TOAST. Trial of Org 10172 in Acute Stroke Treatment. Stroke. 1993;24:35-41.

10. Tu WJ, Zeng XW, Deng A, et al. Circulating FABP4 (fatty acid-binding protein 4) is a novel prognostic biomarker in patients with acute ischemic stroke. Stroke. 2017;48:1531-8.

11. Jia Y, Luo X, Ji Y, et al. Circulating CTRP9 levels are increased in patients with newly diagnosed type 2 diabetes and correlated with insulin resistance. Diabetes Res Clin Pract. 2017;131:116-23.

12. Rasooli Tehrani A, Gholipour S, Sharifi R, et al. Plasma levels of CTRP-3, CTRP-9 and apelin in women with multiple sclerosis. J Neuroimmunol. 2019;333:576968.

13. Wang Z, Wang R, Li Y, et al. Plasma neurofilament light chain as a predictive biomarker for post-stroke cognitive impairment: a prospective cohort study. Front Aging Neurosci. 2021;13:631738.

14. Altamura C, Squitti R, Pasqualetti P, et al. Ceruloplasmin/transferrin system is related to clinical status in acute stroke. Stroke. 2009;40:1282-8.

15. Zhong C, Wang G, Xu T, et al. Tissue inhibitor metalloproteinase- 1 and clinical outcomes after acute ischemic stroke. Neurology. 2019;93: e1675-85.

16. Yang $\mathrm{P}, \mathrm{Zhu} \mathrm{Z}$, Zang $\mathrm{Y}$, et al. Increased serum complement C3 levels are associated with adverse clinical outcomes after ischemic stroke. Stroke. 2021;52:868-77.

17. Wong GW, Krawczyk SA, Kitidis-Mitrokostas C, et al. Identification and characterization of CTRP9, a novel secreted glycoprotein, from adipose tissue that reduces serum glucose in mice and forms heterotrimers with adiponectin. FASEB J. 2009;23: 241-58.

18. Yang G, Song Q, Sun C, et al. Ctrp9 and adiponectin receptors in Nile tilapia (Oreochromis niloticus): molecular cloning, tissue distribution and effects on reproductive genes. Gen Comp Endocrinol. 2018;265:160-73.

19. Selwaness M, Bos D, van den Bouwhuijsen Q, et al. Carotid atherosclerotic plaque characteristics on magnetic resonance imaging relate with history of stroke and coronary heart disease. Stroke. 2016;47: 1542-7.

20. Powers WJ, Rabinstein AA, Ackerson T, et al. 2018 guidelines for the early management of patients with acute ischemic stroke: a guideline for Healthcare Professionals from the American Heart Association/American Stroke Association. Stroke. 2018;49:e46-110.
21. Valgimigli M, Bueno H, Byrne RA, et al. 2017 ESC focused update on dual antiplatelet therapy in coronary artery disease developed in collaboration with EACTS: the Task Force for dual antiplatelet therapy in coronary artery disease of the European Society of Cardiology (ESC) and of the European Association for Cardio-Thoracic Surgery (EACTS). Eur Heart J. 2018;39:213-60.

22. Manninen $\mathrm{V}$, Tenkanen $\mathrm{L}$, Koskinen $\mathrm{P}$, et al. Joint effects of serum triglyceride and LDL cholesterol and HDL cholesterol concentrations on coronary heart disease risk in the Helsinki Heart Study. Implications for treatment. Circulation. 1992;85: 37-45.

23. Wolf RM, Steele KE, Peterson LA, et al. C1q/TNFrelated protein-9 (CTRP9) levels are associated with obesity and decrease following weight loss surgery. J Clin Endocrinol Metab. 2016;101:2211-7.

24. Hwang YC, Woo OS, Park SW, Park CY. Association of serum C1q/TNF-related protein-9 (CTRP9) concentration with visceral adiposity and metabolic syndrome in humans. Int J Obes (Lond). 2014;38: 1207-12.

25. Jung $\mathrm{CH}$, Lee MJ, Kang $\mathrm{YM}$, et al. Association of serum C1q/TNF-related protein-9 concentration with arterial stiffness in subjects with type 2 diabetes. J Clin Endocrinol Metab. 2014;99:E24772484 .

26. Uemura Y, Shibata R, Ohashi K, et al. Adiposederived factor CTRP9 attenuates vascular smooth muscle cell proliferation and neointimal formation. FASEB J. 2013;27:25-33.

27. Wang J, Hang T, Cheng XM, et al. Associations of C1q/TNF-related protein-9 levels in serum and epicardial adipose tissue with coronary atherosclerosis in humans. Biomed Res Int. 2015;2015: 971683.

28. Mantilla-Garcia D, Mourand I, Gascou G, et al. Diffusion weighted imaging and time in acute ischemic stroke, is there any relation? J Neuroradiol. 2017;44:353-60.

29. Banerjee C, Turan TN. Large artery atherosclerosis: extracranial and intracranial. Semin Neurol. 2017;37:307-15.

30. Yiin GS, Howard DP, Paul NL, et al. Age-specific incidence, outcome, cost, and projected future burden of atrial fibrillation-related embolic vascular events: a population-based study. Circulation. 2014;130:1236-44.

31. Shrestha S, Coy S, Bekelis K. Oral antiplatelet and anticoagulant agents in the prevention and 
management of ischemic stroke. Curr Pharm Des. 2017;23:1377-91.

32. Boehme AK, Esenwa C, Elkind MS. Stroke risk factors, genetics, and prevention. Circ Res. 2017;120: 472-95.

33. Sonderer J, Katan KM. Aetiological blood biomarkers of ischaemic stroke. Swiss Med Wkly. 2015;145: w14138.

34. Llombart V, Antolin-Fontes A, Bustamante A, et al. B-type natriuretic peptides help in cardioembolic stroke diagnosis: pooled data meta-analysis. Stroke. 2015;46:1187-95.

35. Katan M, Moon YP, Paik MC, et al. Lipoproteinassociated phospholipase A2 is associated with atherosclerotic stroke risk: the Northern Manhattan Study. PLoS ONE. 2014;9:e83393.
36. Sharp FR, Jickling GC, Stamova B, et al. Molecular markers and mechanisms of stroke: RNA studies of blood in animals and humans. J Cereb Blood Flow Metab. 2011;31:1513-31.

37. Warfarin versus aspirin for prevention of thromboembolism in atrial fibrillation: Stroke prevention in Atrial Fibrillation II Study. Lancet. 1994;343: 687-691.

38. Homma S, Thompson JL, Pullicino PM, et al. Warfarin and aspirin in patients with heart failure and sinus rhythm. N Engl J Med. 2012;366:1859-69.

39. Makris K, Haliassos A, Chondrogianni M, Tsivgoulis G. Blood biomarkers in ischemic stroke: potential role and challenges in clinical practice and research. Crit Rev Clin Lab Sci. 2018;55:294-328. 